\title{
Bibliographie : études génétiques, éditions, manuscrits
}

Janvier 2010-novembre 2010

Danièle Maïsetti et Martine Mesureur-Ceyrat

\section{OpenEdition}

\section{Journals}

Édition électronique

URL : http://journals.openedition.org/genesis/624

DOI : $10.4000 /$ genesis. 624

ISSN : 2268-1590

Éditeur :

Presses universitaires de Paris Sorbonne (PUPS), Société internationale de génétique artistique littéraire et scientifique (SIGALES)

\section{Édition imprimée}

Date de publication : 1 janvier 2011

Pagination : 233-238

ISBN : 978-2-84050-749-9

ISSN : $1167-5101$

Référence électronique

Danièle Maïsetti et Martine Mesureur-Ceyrat, « Bibliographie : études génétiques, éditions, manuscrits », Genesis [En ligne], 32 | 2011, mis en ligne le 22 février 2013, consulté le 02 mai 2019. URL : http:// journals.openedition.org/genesis/624; DOI : 10.4000/genesis.624 


\title{
Bibliographie : études génétiques, éditions, manuscrits
} Janvier 2010-novembre 2010

\author{
Danièle Maïsetti et Martine Mesureur-Ceyrat
}

Cette bibliographie rend compte des publications recensées entre janvier 2010 et novembre 2010. Pour la période antérieure, vous pouvez consulter les références sur le site de l'ITEM, à l'adresse suivante : <www.item.ens.fr/>. Quelques travaux collectifs, ouvrages ou périodiques, ont été intégrés à la toute dernière minute et n'ont par conséquent pu faire l'objet d'un dépouillement : les articles pertinents seront mentionnés dans la prochaine bibliographie. En outre, certaines publications ne nous sont pas encore parvenues, ou ont pu, en dépit de nos efforts, échapper à notre vigilance. C'est pourquoi nous tenons à remercier très chaleureusement celles et ceux qui prennent la peine, non seulement de nous transmettre régulièrement les informations dont ils disposent, mais aussi de nous faire parvenir les articles ou les ouvrages dont ils sont les auteurs.

\section{ÉDITIONS DE TEXTES ET DE MANUSCRITS}

BALZAC Ève de

«Ève de Balzac \& George Sand. Correspondance 1853, textes établis, présentés et annotés par Roger Pierrot et Hervé Yon », dans Mon cher George. Balzac et Sand, histoire d'une amitié. Ouvrage publié à l'occasion de l' exposition Mon cher George. Balzac et Sand, présentée par le musée Balzac à Saché, du 24 mars au 20 juin 2010, Paris, Gallimard, coll. « Lieux et écrivains », 2010, p. 159-165.

Balzac Honoré de, Sand George

«Balzac \& George Sand. Correspondance 1831-1844, textes établis, présentés et annotés par Roger Pierrot et Hervé Yon », dans Mon cher George. Balzac et Sand, histoire d'une amitié. Ouvrage publié à l'occasion de l'exposition Mon cher George. Balzac et Sand, présentée par le musée Balzac à Saché, du 24 mars au 20 juin 2010, Paris, Gallimard, coll. « Lieux et écrivains », 2010, p. 101-158.

BARTHES Roland

Le Lexique de l'auteur. Séminaire à l'École pratique des hautes études 1973-1974, suivi de fragments inédits du Roland Barthes par Roland Barthes, avant-propos d'Éric Marty, présentation et édition d'Anne Herschberg Pierrot, Paris, Éditions du Seuil, coll. « Traces écrites», 2010, $422 \mathrm{p}$

« Roland Barthes, "Phrase - Modernité". Fac-similés et transcriptions [établies par Arlette Attali] de l'inédit, précédés d'un commentaire par Éric Marty, d'une note génétique par Daniel Ferrer et d'une note codicologique par Arlette Attali », Genesis. Manuscrits, recherche, invention, $\mathrm{n}^{\circ} 30$, "Théorie : état des lieux », 2010, p. 239-284.

Delacroix Eugène

Eugène Delacroix. Journal. Tome I (1822-1857) et tome II (18581863), nouvelle édition intégrale établie par Michèle Hannoosh, Paris, José Corti, coll. « Domaine romantique », 2009, 2519 p.
EISENSTEIN Sergueï Mikhaïlovitch

Cinématisme - Peinture et cinéma, édité par Alexandre Laumonier, introduction, notes et commentaires par François Albera, traductions de Valérie Pozner, Elena Rolland, Anne Zouboff, Danièle Huillet, François Albera, Dijon, Les Presses du réel, coll. « Fabula », 2009, 254 p.

Glass House. Du projet de film au film comme projet, édité par Alexandre Laumonier, introduction à Glass House et Destruction de la forme et transparence par François Albera, traductions de Valérie Pozner, Michail Maiatsky et François Albera, Dijon, Les Presses du réel, coll. «Fabula », 2009, 101 p.

LEVINAs Emmanuel

Euvres 1. Carnets de captivité suivi de Écrits sur la captivité et Notes philosophiques diverses, volume publié sous la responsabilité de Rodolphe Calin et de Catherine Chalier, établissement du texte, annotation matérielle, avertissement par Rodophe Calin, préface et notes explicatives par Rodophe Calin et Catherine Chalier, préface générale de Jean-Luc Marion de l'Académie française, Paris, Bernard Grasset/ IMEC, 2009, 499 p.

\section{OUVRAGES}

CAlvet Louis-Jean

Le Jeu du signe, Paris, Éditions du Seuil, coll. « Fiction \& Cie, », 2010, $197 \mathrm{p}$.

GiURIATo Davide

Mikrographien. Zu einer Poetologie des Schreibens in Walter Benjamins Kindheitserinnerungen (1932-1939), München, Wilhelm Fink, coll. «Zur Genealogie des Schreibens, 5 », 2006, 324 p.

Hurlebusch Klaus

Buchstabe und Geist, Geist und Buchstabe. Arbeiten zur Editionsphilologie, Frankfurt, Berlin, Peter Lang, coll. « Hamburger Beiträge zur Editionsphilologie, 50 », 2010, 380 p. 
LAHIRE Bernard

Franz Kafka : éléments pour une théorie de la création littéraire, Paris, Éditions La Découverte, coll. «Textes à l'appui/laboratoire des sciences sociales », 2010, $632 \mathrm{p}$.

\section{Morim De Carvalho Edmundo}

Poésie et science chez Bachelard, Paris, Éditions L'Harmattan, 2010, $294 \mathrm{p}$.

RaITT Alan W.

Flaubert's First Novel. A Study of the 1845 Éducation sentimentale, Oxford/Bern/Berlin, Peter Lang, coll. « Le Romantisme et après en France/Romanticism and after in France, $18 », 2010,134$ p.

SugAYA Norioki

Flaubert épistémologue. Autour du dossier médical de Bouvard et Pécuchet, Amsterdam/New York, Rodopi, coll. « Faux titre, 346 », 2010, $276 \mathrm{p}$.

Yoshikawa Kazuyoshi

Proust et l'art pictural, Paris, Honoré Champion, coll. « Recherches proustiennes, $14 », 2010,409 \mathrm{p}$.

\section{OUVRAGES COLLECTIFS}

Anfangen zu schreiben. Ein kardinales Moment von Textgenese und Schreibprozess im literarischen Archiv des 20. Jahrhunderts, herausgegeben von Hubert Thüring, Corinna Jäger-Trees und Michael Schläfli, München, Wilhelm Fink, coll. «Zur Genealogie des Schreibens, $11 », 2009,345$ p.

La Chambre noire d'André Gide, sous la direction d'Alain Goulet [Actes du colloque André Gide, BnF, 9 octobre 2009], Paris, Éditions Le Manuscrit, coll. « Recherche Université », 2009, 192 p.

Criação em debate, Claudia Amigo Pino organizadora, São Paulo, Humanitas, 2007, 301 p.

Flaubert et la peinture, textes réunis et présentés par Gisèle Séginger, Paris, Lettres modernes Minard, coll. «Gustave Flaubert, 7 », 2010, 337 p.

Genèses théâtrales, dir. Almuth Grésillon, Marie-Madeleine MervantRoux, Dominique Budor, Paris, CNRS Éditions, coll. « Textes et Manuscrits », 2010, 272 p. (avec un cahier couleur de 32 p.).

Konjektur und Krux. Zur Methodenpolitik der Philologie, herausgegeben von Anne Bohnenkamp, Kai Bremer, Uwe Wirth, Irmgard M. Wirtz, Göttingen, Wallstein Verlag, 2010, 420 p.

Linguistique et littérature : Cluny, 40 ans après, dir. Driss Ablali et Margareta Kastberg Sjöblom [Actes du colloque international, 29-31 octobre 2007, Besançon], Besançon, Presses universitaires de Franche-Comté, coll. «Annales littéraires de l'université de FrancheComté, 863 », 2010, 344 p.

Littératures au Sud, dir. Marc Cheymol, préface de Bernard Cerquiglini, postface de Souleymane Bachir Diagne, Paris, Éditions des archives contemporaines/Agence universitaire de la Francophonie, coll. «Actualité scientifique », 2009, 254 p.
Originalités proustiennes, dir. Philippe Chardin, Paris, Éditions Kimé, 2010, 297 p.

Les Reformulations pluri-sémiotiques en contexte de formation, dir. Alain Rabatel, Besançon, Presses universitaires de Franche-Comté, coll. « Annales littéraires de 1'université de Franche-Comté, 864 », 2010, 298 p.

Savoirs en récit. I, Flaubert : la politique, l'art, l' histoire, textes réunis et présentés par Anne Herschberg Pierrot, Saint-Denis, PUV, coll. « Manuscrits Modernes », 2010, 180 p.

Savoirs en récit. II, Éclats de savoirs : Balzac, Nerval, Flaubert, Verne, Les Goncourt, textes réunis et présentés par Jacques Neefs, SaintDenis, PUV, coll. « Manuscrits Modernes », 2010, 166 p.

Schreibprozesse, herausgegeben von Peter Hughes, Thomas Fries, Tan Wälchli, München, Wilhelm Fink, coll. «Zur Genealogie des Schreibens, 7 », 2008, 325 p.

Texte zur modernen Philologie, herausgegeben von Kai Bremer et Uwe Wirth, Stuttgart, Philipp Reclam jun., 2010, 335 p.

\section{ARTICLES ET CONTRIBUTIONS À DES OUVRAGES COLLECTIFS ET DES REVUES}

Alamargot Denis, Lebrave Jean-Louis

«The study of professional writing. A joint contribution from cognitive psychology and genetic criticism », European psychologist, vol. XV, $\mathrm{n}^{\circ} 1,2010$, p. $12-22$.

Amrein Ursula

«Verschriftete Bilder : Gottfried Kellers Bildpoetik im Prozess der Säkularisierung », dans Schreibprozesse, München, Wilhelm Fink, coll. «Zur Genealogie des Schreibens, 7 », 2008, p. 51-76.

ANDRÉ Julie

«Plan, scénarios, résumés : hypothèses sur la construction du Cahier $46 »$, Bulletin d'informations proustiennes, $\mathrm{n}^{\circ} 40,2010$, p. 31-44.

Artières Philippe

«Pour une histoire sociale de l'écriture. Histoire et génétique textuelle », Genesis. Manuscrits, recherche, invention, ${ }^{\circ} 30$ «Théorie : état des lieux », 2010, p. 185-189.

Biasi Pierre-Marc de

«Pour une génétique généralisée : l'approche des processus à l'âge numérique », Genesis. Manuscrits, recherche, invention, $\mathrm{n}^{\circ} 30$, « Théorie : état des lieux », 2010, p. 163-175.

«Le projet de l'équipe "Manuscrit francophone" de l'ITEM », dans Littératures au Sud, Paris, Éditions des archives contemporaines/ Agence universitaire de la Francophonie, coll. «Actualité scientifique », 2009, p. 159-161.

BISANG Regula

«To Show or Not to Show : Stage Directions, Textual History, and the Play Scene in Shakespeare's Hamlet », dans Schreibprozesse, München, Wilhelm Fink, coll. «Zur Genealogie des Schreibens, 7 », 2008, p. $15-31$. 


\section{BoIE Bernhild}

«Auf unsteten Bahnen. Anfänge des Schreibens bei Georg Heym, Günter Eich und Peter Rühmkorf », dans Anfangen zu schreiben. Ein kardinales Moment von Textgenese und Schreibprozess im literarischen Archiv des 20. Jahrhunderts, München, Wilhelm Fink, coll. «Zur Genealogie des Schreibens, $11 », 2009$, p. 237-254.

\section{Boulez Pierre}

«Écriture musicale et accident, entretien avec Dominique Jameux », Genesis. Manuscrits, recherche, invention, $\mathrm{n}^{\circ} 30$, « Théorie : état des lieux », 2010, p. 219-228.

\section{Bourdin Béatrice, Cogis Danièle, Foulin Jean-Noël}

«Influence des traitements graphomoteurs et orthographiques sur la production de textes écrits : perspective pluridisciplinaire », Langages, $\mathrm{n}^{\circ} 177$, «Traitement des contraintes de la production d'écrits : aspects linguistiques et psycholinguistiques », 2010, p. 57-82

CAVAILlès Nicolas

«Génétique et dialogisme », Genesis. Manuscrits, recherche, invention, $\mathrm{n}^{\circ}$ 30, « Théorie : état des lieux », 2010, p. 191-195.

Cerquiglini Bernard

«Vingt ans après », Genesis. Manuscrits, recherche, invention, $\mathrm{n}^{\circ} 30$, «Théorie : état des lieux », 2010, p. 15-17.

\section{Chuy Maria, Rondelli Fabienne}

«Traitement des contraintes linguistiques et cognitives dans la construction de la cohérence textuelle », Langages, $\mathrm{n}^{\circ} 177$, « Traitement des contraintes de la production d'écrits : aspects linguistiques et psycholinguistiques », 2010, p. 83-111.

Colla Fernando

«Les Archives Virtuelles Latino-Américaines : héritages et perspectives », Escritural. Écritures d'Amérique latine, $\mathrm{n}^{\circ} 1$, «1. Neruda : discurso poético, escritura e intertextualidad ; 2. Les Archives Virtuelles Latino-Américaines », 2009, p. 399-401.

Compagnon Antoine

«Génétique, intertextualité et histoire littéraire, entretien avec PierreMarc de Biasi et Anne Herschberg Pierrot », Genesis. Manuscrits, recherche, invention, $\mathrm{n}^{\circ} 30$, « Théorie : état des lieux », 2010, p. 55-57.

Crasson Aurèle

«Archives manuscrites littéraires : l'apport du numérique pour l'édition et la recherche scientifique », Genesis. Manuscrits, recherche, invention, $\mathrm{n}^{\circ} 30$, « Théorie : état des lieux », 2010, p. 43-47.

DEBRU Claude

«"Penser avec les mains" : critique génétique et épistémologie, entretien avec Jean-Louis Lebrave », Genesis. Manuscrits, recherche, invention, $\mathrm{n}^{\circ} 30$, «Théorie : état des lieux », 2010, p. 229-234.

D'IORIO Paolo

«Qu'est-ce qu'une édition génétique numérique ? ", Genesis Manuscrits, recherche, invention, $\mathrm{n}^{\circ} 30$, «Théorie : état des lieux », 2010, p. 49-53.

\section{Doquet-Lacoste Claire}

«Postures énonciatives et marqueurs de reformulation en situation de conférence avec diaporama. Alors, effectivement, donc », dans Les Reformulations pluri-sémiotiques en contexte de formation, Besançon, Presses universitaires de Franche-Comté, coll. «Annales littéraires de l'université de Franche-Comté, 864 », 2010, p. 47-71.

DORD-Crouslé Stéphanie

«C.R. Éric Le Calvez : Genèses flaubertiennes, Amsterdam/New York, Rodopi, 2009 », Revue d'Histoire littéraire de la France, 110e année, $\mathrm{n}^{\circ} 1$, mars 2010, p. 223-224.

Dord-Crouslé Stéphanie, Mercier Félicie

« Les notes prises par Flaubert sur la revue L'Artiste », dans Flaubert et la peinture, Paris, Lettres modernes Minard, coll. « Gustave Flaubert, 7 », 2010, p. 255-309.

ESPAGNE Michel

«Philologie et critique génétique », Genesis. Manuscrits, recherche, invention, $\mathrm{n}^{\circ} 30$, «Théorie : état des lieux », 2010, p. 19-20.

\section{FEITKNECHT Thomas}

" „Um meine Geschichte zu erzählen, muss ich weit vorn anfangen“ Hesses Schreiben im Zirkel von Krise und Therapie », dans Anfangen zu schreiben. Ein kardinales Moment von Textgenese und Schreibprozess im literarischen Archiv des 20. Jahrhundert, München, Wilhelm Fink, coll. «Zur Genealogie des Schreibens, $11 », 2009$, p. 257-270.

FERRER Daniel

« Critique génétique et philologie : racine de la différence », Genesis. Manuscrits, recherche, invention, $\mathrm{n}^{\circ} 30$, « Théorie : état des lieux », 2010, p. 21-23

«Mondes possibles, mondes fictionnels, mondes construits et processus de genèse ", Genesis. Manuscrits, recherche, invention, $\mathrm{n}^{\circ} 30$, « Théorie : état des lieux », 2010, p. 109-130.

\section{GABLER Hans Walter}

«Les livres, les textes et la critique », Genesis. Manuscrits, recherche, invention, $\mathrm{n}^{\circ} 30$, « Théorie : état des lieux », 2010, p. 41-42.

Germain Marie Odile

«Sur quelques manuscrits de Gide à la Bibliothèque nationale de France », dans La Chambre noire d'André Gide, Paris, Éditions Le Manuscrit, coll. « Recherche Université », 2009, p. 155-164.

\section{Goujon Francine}

«Sodome et Gomorrhe I : un lieu racinien », dans Originalités proustiennes, Paris, Éditions Kimé, 2010, p. 161-174.

Goulet Alain

" "Peut-être même approche-t-on de plus près la vérité dans le roman" », dans La Chambre noire d'André Gide, Paris, Éditions Le Manuscrit, coll. « Recherche Université », 2009, p. 65-102.

GRODDECK Wolfram

" „Ich schreibe hier...“ Textgenese im Text. Zu Robert Walsers Prosastück Die leichte Hochachtung », dans Anfangen zu schreiben. Ein kardinales Moment von Textgenese und Schreibprozess im literarischen Archiv des 20. Jahrhunderts, München, Wilhelm Fink, coll. «Zur Genealogie des Schreibens, 11 », 2009, p. 97-108. 


\section{HAMON Philippe}

«Quelques questions à la génétique », Genesis. Manuscrits, recherche, invention, invention, $\mathrm{n}^{\circ} 30$, « Théorie : état des lieux », 2010, p. 65-68.

HAY Louis

"Ce que dit Genesis. Hommage à Almuth Grésillon», Genesis. Manuscrits, recherche, invention, $\mathrm{n}^{\circ} 30$, «Théorie : état des lieux », 2010, p. 11-14.

\section{HenKe Silvia}

«Hermann Burger : Hokuspokus. Abracadabra. Simsalabim. Oder wie aus nichts etwas anfängt », dans Anfangen zu schreiben. Ein kardinales Moment von Textgenese und Schreibprozess im literarischen Archiv des 20. Jahrhunderts, München, Wilhelm Fink, coll. « Zur Genealogie des Schreibens, $11 », 2009$, p. 109-126.

Herschberg Pierrot Anne

«Approches génétiques du style », dans Stylistiques ?, dir. Laurence Bougault, Rennes, PUR, coll. « interférences », 2010, p. 33-40.

"Chemins de l'œuvre ", Genesis. Manuscrits, recherche, invention, n³0, « Théorie : état des lieux », 2010, p. 87-108.

"Style de genèse et style d'auteur », Romantisme, vol. II, $\mathrm{n}^{\circ}$ 148, juin 2010, p. 103-113.

HoNOLD Alexander

«Initiationen und Initialen. Franz Kafka in seinen Oktavheften », dans Anfangen zu schreiben. Ein kardinales Moment von Textgenese und Schreibprozess im literarischen Archiv des 20. Jahrhunderts, München, Wilhelm Fink, coll. «Zur Genealogie des Schreibens, 11 », 2009, p. 77-96.

Hughes Peter

"Coleridge's Unwriting », dans Schreibprozesse, München, Wilhelm Fink, coll. «Zur Genealogie des Schreibens, 7 », 2008, p. 35-48.

JäGER-Trees Corinna

«Zu Otto F. Walters Schreibkonzept des Anfangens in Der Stumme und Zeit des Fasans », dans Anfangen zu schreiben. Ein kardinales Moment von Textgenese und Schreibprozess im literarischen Archiv des 20. Jahrhunderts, München, Wilhelm Fink, coll. « Zur Genealogie des Schreibens, $11 », 2009$, p. 271-290.

KAMMER Stephan

«Zettelkasten und bewegliche Lettern. Die poetologische Entzauberung des Anfang(en)s », dans Anfangen zu schreiben. Ein kardinales Moment von Textgenese und Schreibprozess im literarischen Archiv des 20. Jahrhunderts, München, Wilhelm Fink, coll. «Zur Genealogie des Schreibens, $11 », 2009$, p. 29-42.

\section{KASPER Monika}

«Vom Malen zum Schreiben : Gottfried Kellers Berliner Schreibunterlage », dans Schreibprozesse, München, Wilhelm Fink, coll. « Zur Genealogie des Schreibens, 7 », 2008, p. 77-99.

Keller Luzius

«Inventio, dispositio und elocutio bei Marcel Proust : Die Erfindung der „Petites Madeleines“ als Schreibprozess », dans Schreibprozesse, München, Wilhelm Fink, coll. «Zur Genealogie des Schreibens, 7 », 2008, p. 159-177.

\section{LEBRAVE Jean-Louis}

«La critique génétique et les sciences cognitives », Genesis. Manuscrits, recherche, invention, $\mathrm{n}^{\circ} 30$, « Théorie : état des lieux », 2010, p. 131-150

LERICHE Françoise

«Fluctuation des notions d'“originalité" et d"“artiste original" dans la genèse du roman proustien », dans Originalités proustiennes, Paris, Éditions Kimé, 2010, p. 117-131.

LoIs Elida

« À propos du rapport entre les archives documentaires et la réflexion théorique », Escritural. Écritures d'Amérique latine, $\mathrm{n}^{\circ} 1$, «1. Neruda : discurso poético, escritura e intertextualidad ; 2. Les Archives Virtuelles Latino-Américaines », 2009, p. 402-405.

Lumbroso Olivier

«Pour une didactique du prérédactionnel », Genesis. Manuscrits, recherche, invention, $\mathrm{n}^{\circ} 30$, « Théorie : état des lieux », 2010 p. 177-184.

\section{LÜTHI Ariane}

« La note-amorce. Une lecture des Papiers collés de Georges Peros », dans Schreibprozesse, München, Wilhelm Fink, coll. «Zur Genealogie des Schreibens, 7 », 2008, p. 265-286.

MARTY Éric

«Roland Barthes, "Phrase - Modernité" : une conférence sur la phrase. Présentation du dossier inédit ", Genesis. Manuscrits, recherche, invention, $\mathrm{n}^{\circ} 30$, « Théorie : état des lieux », 2010, p. 237-238.

\section{Masson Pierre}

« Histoires de portes et de chambres », dans La Chambre noire d'André Gide, Paris, Éditions Le Manuscrit, coll. « Recherche Université », 2009 , p. $39-64$

MaUriac Dyer Nathalie

« Memnon barométrique, ou Spleen à la manière de Proust », dans Originalités proustiennes, Paris, Éditions Kimé, 2010, p. 185-195.

\section{MitTerand Henri}

"Sur le "scénarique" ", Genesis. Manuscrits, recherche, invention, $\mathrm{n}^{\circ} 30$, « Théorie : état des lieux », 2010, p. 69-85.

«Critique génétique et sociocritique, entretien avec Pierre-Marc de Biasi et Anne Herschberg Pierrot », Genesis. Manuscrits, recherche, invention, $\mathrm{n}^{\circ} 30$, « Théorie : état des lieux », 2010, p. 59-63.

NATUREL Mireille

«Déconstruire pour mieux reconstruire les rapports nouveaux entre les choses », dans Originalités proustiennes, Paris, Éditions Kimé, 2010 p. $95-103$.

NAKANO Chizu

"Proust lecteur de Henry Bordeaux : Sainte-Beuve retrouvé ?», Bulletin d'informations proustiennes, $\mathrm{n}^{\circ} 40,2010$, p. 139-150.

\section{Ogane Atsuko}

«Critique génétique d'Hérodias de Flaubert : la formation de la première danse [article en japonais] », Journal of Arts and sciences, $\mathrm{n}^{\circ} 20$, January 2010, p. 1-25. 
Olive Thierry, Passerault Jean-Michel, Lebrave Jean-Louis,

LE Bigot Nathalie

« La dimension visuo-spatiale de la production des textes : approches de psychologie cognitive et de critique génétique », Langages, $\mathrm{n}^{\circ} 177$, «Traitement des contraintes de la production d'écrits : aspects linguistiques et psycholinguistiques », 2010, p. 29-55.

Paolacci Véronique, Favart Monique

« Traitement des marques de cohésion par les jeunes scripteurs : 1'utilisation de la ponctuation et des connecteurs à l'entrée en sixième. Approche linguistique, cognitive et didactique », Langages, $\mathrm{n}^{\circ} 177$, «Traitement des contraintes de la production d'écrits : aspects linguistiques et psycholinguistiques », 2010, p. 113-128.

\section{PÉTILlon Sabine}

« Le manuscrit d'écrivain, terrain d'investigations linguistiques de la production écrite. Esquisse d'un panorama », dans Linguistique et littérature : Cluny, 40 ans après, Besançon, Presses universitaires de Franche-Comté, coll. «Annales littéraires de l'université de FrancheComté, 863 », 2010, p. 293-304.

«Psychologie cognitive et production écrite : de l'effet "épistémique" à la catalyse », Genesis. Manuscrits, recherche, invention, $\mathrm{n}^{\circ} 30$, «Théorie : état des lieux », 2010, p. 197-206.

Plane Sylvie, Alamargot Denis, Lebrave Jean-Louis

« Temporalité de l'écriture et rôle du texte produit dans l'activité rédactionnelle », Langages, $\mathrm{n}^{\circ} 177$ « Traitement des contraintes de la production d'écrits : aspects linguistiques et psycholinguistiques », 2010, p. 7-28.

Plane Sylvie, Olive Thierry, Alamargot Denis

«Présentation : pour une approche pluridisciplinaire des contraintes de la production écrite », Langages, $\mathrm{n}^{\circ} 177$ « Traitement des contraintes de la production d'écrits : aspects linguistiques et psycholinguistiques », 2010, p. 3-5.

ReIBNITZ Barbara von

« Komma überschreibt Punkt. Anfangen und Nicht-Aufhören(können) in Robert Walsers Romanerstling Geschwister Tanner », dans Anfangen zu schreiben. Ein kardinales Moment von Textgenese und Schreibprozess im literarischen Archiv des 20. Jahrhunderts, München, Wilhelm Fink, coll. «Zur Genealogie des Schreibens, 11 », 2009, p. 129-146.

Rico Francisco

«Éditions savantes et lecteurs réels », Genesis. Manuscrits, recherche invention, $\mathrm{n}^{\circ} 30$, « Théorie : état des lieux », 2010, p. 29-34.

\section{RIFFARD Claire}

«Un exemple de valorisation des manuscrits francophones : sauvegarde et valorisation des manuscrits malgaches », dans Littératures au Sud, Paris, Éditions des archives contemporaines/Agence universitaire de la Francophonie, coll. «Actualité scientifique », 2009, p. 163-166.

Röllin Beat, Haase Marie-Luise, Stockmar René, Trenkle Franziska

« „Der späte Nietzsche“ - Schreibprozess und Heftedition », dans Schreibprozesse, München, Wilhelm Fink, coll. « Zur Genealogie des Schreibens, 7 », 2008, p. 103-115.

\section{SAGAert Martine}

«Présentation du DVD-ROM André Gide l'écriture vive », dans La Chambre noire d'André Gide, Paris, Éditions Le Manuscrit, coll. « Recherche Université », 2009, p. 165-181.

SALles Cecilia Almeida

«Réseaux de la création : construction de l'œuvre d'art », Genesis. Manuscrits, recherche, invention, $\mathrm{n}^{\circ} 30$, «Théorie : état des lieux », 2010 , p. 207-211.

SCHLÄFLI Michael

« „Der Text kommt aus der Dunkelheit !“ Christoph Geiser schreibt Im Freigehege », dans Anfangen zu schreiben. Ein kardinales Moment von Textgenese und Schreibprozess im literarischen Archiv des 20. Jahrhunderts, München, Wilhelm Fink, coll. «Zur Genealogie des Schreibens, $11 », 2009$, p. 301-324.

Segre Cesare, Giaveri Maria Teresa

«Philologie italienne et critique génétique, entretien avec Maria Teresa Giaveri, en collaboration avec Erica Durante », Genesis. Manuscrits, recherche, invention, $\mathrm{n}^{\circ} 30$, « Théorie : état des lieux », 2010, p. 25-27.

SENN Fritz

« Fiktiver Schreibprozess : Leopold Bloom verfasst einen Brief », dans Schreibprozesse, München, Wilhelm Fink, coll. « Zur Genealogie des Schreibens, 7 », 2008, p. 181-189.

Shillingsburg Peter, EgGert Paul

« Le paysage éditorial anglo-américain de 1980 à 2005 », Genesis. Manuscrits, recherche, invention, $\mathrm{n}^{\circ} 30$, «Théorie : état des lieux », 2010, p. 35-39.

TEYSSANDIER Laurence

«Sur quelques aspects de la genèse de la soirée Verdurin, », Bulletin d'informations proustiennes, $\mathrm{n}^{\circ} 40,2010$, p. 45-54.

THÜRING Hubert

"Anfangen zu schreiben. Einleitung », dans Anfangen zu schreiben. Ein kardinales Moment von Textgenese und Schreibprozess im literarischen Archiv des 20. Jahrhunderts, München, Wilhelm Fink, coll. «Zur Genealogie des Schreibens, $11 », 2009$, p. 9-25.

«Der späte Anfänger Friedrich Glauser », dans Anfangen zu schreiben. Ein kardinales Moment von Textgenese und Schreibprozess im literarischen Archiv des 20. Jahrhunderts, München, Wilhelm Fink, coll. «Zur Genealogie des Schreibens, $11 », 2009$, p. 147-172.

VAN Hulle Dirk

«Espèces d'origine. Darwin et la métaphore biologique dans la critique génétique », Genesis. Manuscrits, recherche, invention, $\mathrm{n}^{\circ} 30$, « Théorie : état des lieux », 2010, p. 151-162.

Viollet Catherine

«À la rencontre des journaux personnels », avant-propos, Cahiers du Monde russe, $\mathrm{n}^{\circ}$ 50/1, «Écrits personnels Russie XVIII -XXe s. », 2010, p. 9-15.

WEBER Ulrich

« Dürrenmatts Fiktion „Das Hirn“ : Textgenese zwischen Schöpfung und Evolution », dans Schreibprozesse, München, Wilhelm Fink, coll. «Zur Genealogie des Schreibens, 7 », 2008, p. 237-261. 


\section{Willemart Philippe}

«Comment comprendre et penser le manuscrit?», Genesis. Manuscrits, recherche, invention, $\mathrm{n}^{\circ} 30$, «Théorie : état des lieux », 2010, p. 213-218.

WIRTz Irmgard

«Elias Canettis Aufzeichnungen. Kein Anfang, kein Ende », dans Anfangen zu schreiben. Ein kardinales Moment von Textgenese und Schreibprozess im literarischen Archiv des 20. Jahrhunderts, München, Wilhelm Fink, coll. «Zur Genealogie des Schreibens, 11 », 2009, p. $173-180$.

Wise Pyra

«Lettres et dédicaces inédites de Proust et de quelques correspondants », Bulletin d' informations proustiennes, ${ }^{\circ} 40,2010$, p. 9-28.

WitTManN Jean-Michel

«Quand l'écrivain remet son ouvrage sur le métier : l'exemple d'une page supprimée de Paludes », dans La Chambre noire d'André Gide, Paris, Éditions Le Manuscrit, coll. « Recherche Université », 2009, p. 19-38.

ZANETTI Sandro

«Wo beginnt der Anfang ? Lektürenotizen - erste Gedichtentwürfe bei Paul Celan », dans Anfangen zu schreiben. Ein kardinales Moment von Textgenese und Schreibprozess im literarischen Archiv des 20. Jahrhunderts, München, Wilhelm Fink, coll. «Zur Genealogie des Schreibens, $11 », 2009$, p. 215-235.

\section{CATALOGUE D'EXPOSITION}

Mon cher George. Balzac et Sand, histoire d'une amitié, ouvrage publié à l'occasion de l'exposition Mon cher George. Balzac et Sand, présentée par le musée Balzac à Saché, du 24 mars au 20 juin 2010, Paris Gallimard, coll. «Lieux et écrivains », 2010, 182 p.

\section{REVUES}

Bulletin d'informations proustiennes, $\mathrm{n}^{\circ} 40,2010$.

Cahiers du Monde russe, $\mathrm{n}^{\circ}$ 50/1, «Écrits personnels », 2010.

Escritural. Écritures d'Amérique latine, $\mathrm{n}^{\circ} 1$, «1. Neruda : discurso poético, escritura e intertextualidad ; 2. Les Archives Virtuelles LatinoAméricaines » [Congrès international organisé par la Maison des Sciences de l'Homme et de la Société de Poitiers, en octobre 2008], dir. Fernando Moreno, 2009.

Genesis. Manuscrits, recherche, invention, $\mathrm{n}^{\circ} 30$, «Théorie : état des lieux », $2010 ; n^{\circ} 31$, « Composer », 2010.

Langages, $\mathrm{n}^{\circ} 177$, «Traitement des contraintes de la production d'écrits : aspects linguistiques et psycholinguistiques », textes réunis par Sylvie Plane, Thierry Olive et Denis Alamargot, 2010.

Manuscritica, $\mathrm{n}^{\circ}$ 17, 2009.

Recherches \& Travaux, $\mathrm{n}^{\circ} 75$, « L'autoportrait fragmentaire », textes réunis par Brigitte Ferrato-Combe, 2010.

\section{PUBLICATIONS ÉLECTRONIQUES}

\section{Revues}

Flaubert. Revue critique et génétique [en ligne], $\mathrm{n}^{\circ}$ 3, «Le désir amoureux », dossier coordonné par Pierre-Marc de Biasi, septembre 2010, $<$ http://flaubert.revues.org/index958.html>.

Recto/Verso, $n^{\circ}$ 6, « Genèse de la pensée. II, Cheminements et procédures », septembre 2010, <http://.revuerectoverso.com/spip. php? page $=$ numero\&id_rubrique $=80>$.

\section{Articles}

\section{BeLlon Guillaume}

«Pour une genèse parlée de la pensée. L'Abécédaire de Gilles Deleuze », Recto/Verso, $\mathrm{n}^{\circ}$ 6, « Genèse de la pensée. II, Cheminements et procédures », septembre 2010, <http://revuerectoverso.com/spip. php?article181>.

Giraud Sylvie

"Hérode Antipas "ne bande plus pr elle" », Flaubert. Revue critique et génétique [en ligne], $\mathrm{n}^{\circ}$ 3, « Le désir amoureux », 30 septembre 2010, <http://flaubert.revues.org/index1183.html>.

Herschberg Pierrot Anne

«Bouvard et Pécuchet et le désir amoureux », Flaubert. Revue critique et génétique [en ligne], $\mathrm{n}^{\circ} 3$, « Le désir amoureux », 30 septembre 2010, <http://flaubert.revues.org/index953.html>.

LEFORT-FAVREAU Julien

«Genèse et postérité du bio-pouvoir. Strates de la pensée, de Foucault à Agamben », Recto/Verso, ${ }^{\circ}$ 6, "Genèse de la pensée. II, Cheminements et procédures », septembre 2010, <http://revuerectoverso.com/spip.php?article183>.

Matsuzawa Kazuhiro

«L'illusion de la désillusion : Essai d'interprétation génétique de L'Éducation sentimentale », Flaubert. Revue critique et génétique [en ligne], $\mathrm{n}^{\circ} 3$, «Le désir amoureux », 30 septembre 2010, <http:// flaubert.revues.org/index $955 . \mathrm{html}>$.

Mondon Geneviève

"Une éducation sentimentale ou le roman d'amour de Salammbô », Flaubert. Revue critique et génétique [en ligne], $\mathrm{n}^{\circ} 3$, « Le désir amoureux », 30 septembre 2010, <http://flaubert.revues.org/index 1161. html>.

Ogane Atsuko

«Hérodias, splendeur du vers et de la prose : le serpent ou la genèse de la femme fatale dans la danse de Salomé », dans Revue Flaubert [en ligne], $\mathrm{n}^{\circ}$ 9, « Flaubert et la confusion des genres, numéro dirigé par Sandra Glatigny, avec la collaboration de Juliette Azoulai », 2009, $<$ http://flaubert.univ-rouen.fr/jet/public/revue/article.php?id=36>.

TestenoIRe Pierre-Yves

"Genèse manuscrite d'un principe saussurien. L'exemple de la linéraité », Recto/Verso, $\mathrm{n}^{\circ} 6$, «Genèse de la pensée. II, Cheminements et procédures », septembre 2010, <http://revuerectoverso.com/spip. php?article179>. 